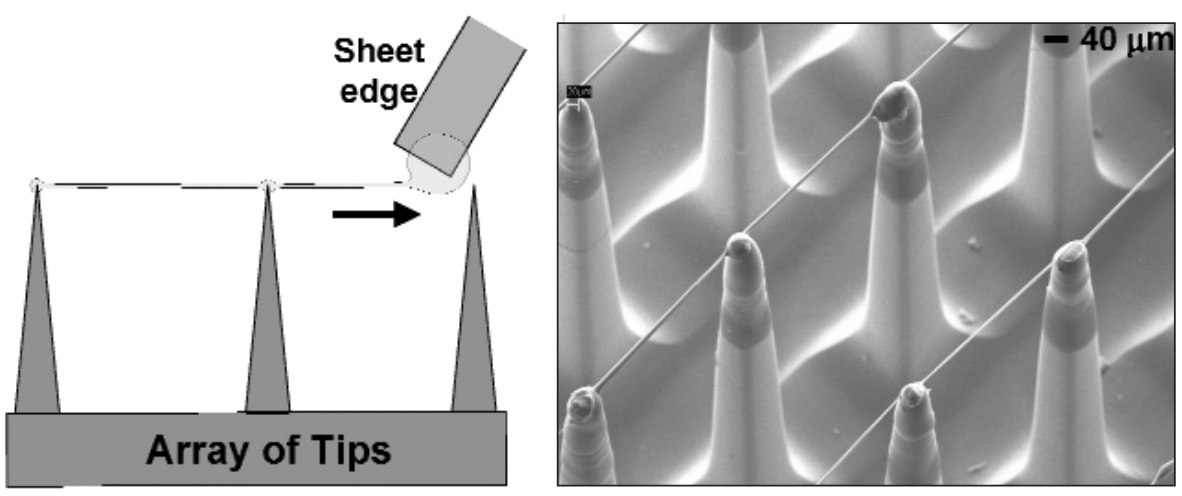

Figure 1. Fabrication of suspended nanostructures by direct drawing of polymer fibers. $A$ drop of polymer is applied at the edge of a sheet and quickly drawn across a tip array creating multiple suspended parallel fibers.

polymer fibers with desired materials using various deposition techniques followed by selective removal of the polymer core. The researchers demonstrated capillaries in chrome, parylene, gold, and glass.
The speed and ease of fabrication by this direct-drawing method compared to conventional multi-step lithography, together with choice of polymer with desirable properties, opens the possibility of applications in the fabrication of microfluidic devices, and optical and sensing elements.

SOMA CHATTOPADHYAY

\section{Use of Centrifuge Force to Fabricate Reticulated Porous Ceramics Results in Uniform Structure}

Reticulated porous ceramics (RPCs) are synthesized materials composed of an arrangement of superimposed ceramic lattices. These materials exhibit porosity higher than $70 \%$. Control of the pore structure is key for application of RPCs. A common method for manufacturing RPCs consists in immersing a polyurethane sponge in prepared ceramic slurry, removing the excess slurry by roll pressing and then sintering. However, the rollpress method does not effectively remove the excess slurry accumulated in the joints of the lattice. An alternative process to remove the excess slurry from the preform by utilizing centrifugal force has been presented by X. Pu, X. Liu, F. Qiu,

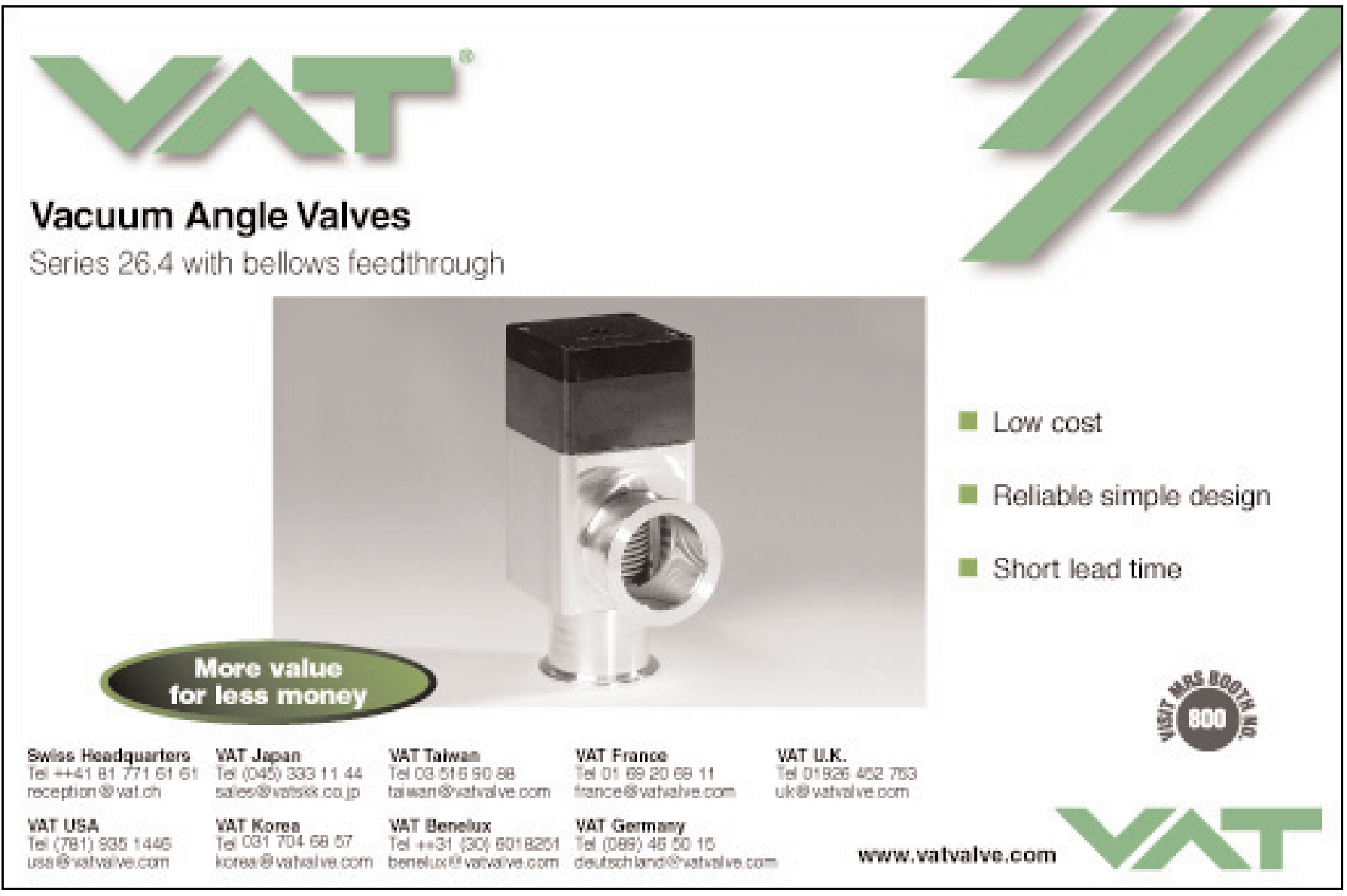




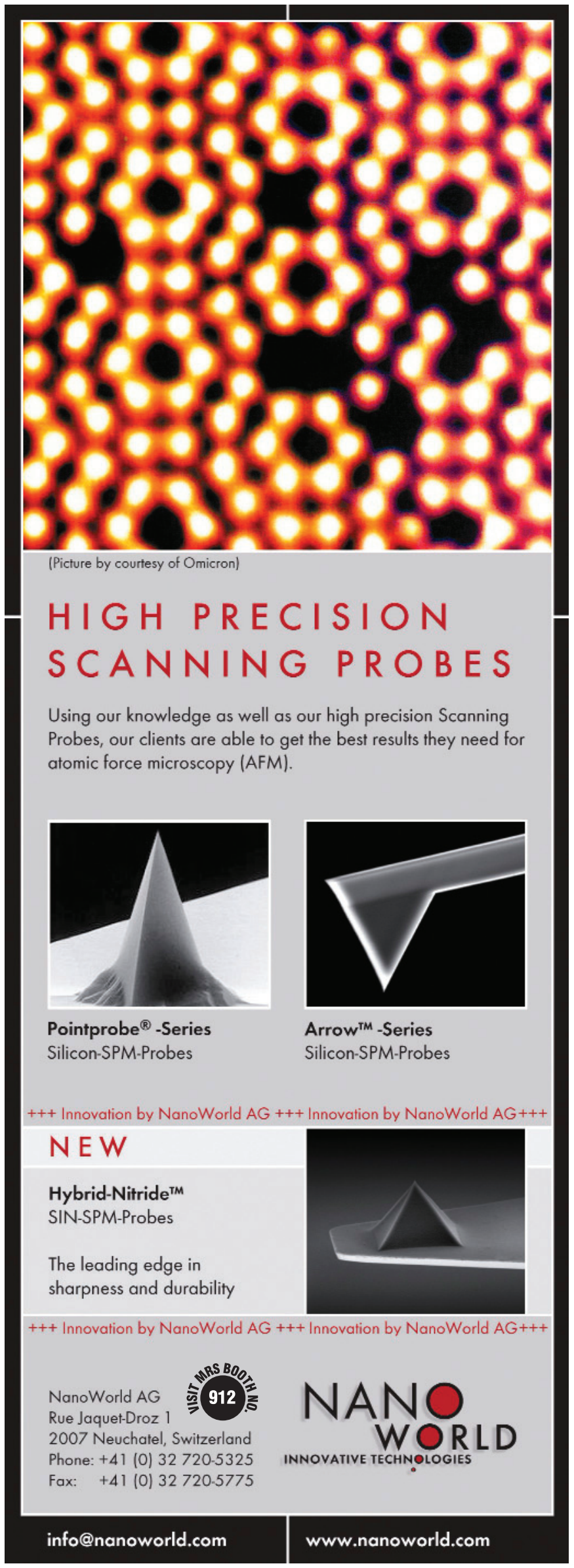

and L. Huang from the Shanghai Institute of Ceramics of the Chinese Academy of Sciences. Their technique ensures a uniform structure on the ceramic reticule.

As reported in the July issue of the Journal of the American Ceramic Society (p. 1392), the researchers prepared a slurry from silicon nitride powders mixed with $7 \mathrm{wt} \%$ alumina added as a sintering additive, $23 \mathrm{wt} \%$ silica sol as a binder, $1.5 \mathrm{wt} \%$ carboxymethylcellulose as a thickening agent, and $0.2 \mathrm{wt} \%$ of Nopco 267-A as an antifoaming agent. The slurry was ball milled using silicon nitride balls. A polyurethane sponge was submerged in slurry and compressed there to achieve the most adsorption possible, then centrifuged at high speed. In this manner, the lattice was uniformly covered with a thin film. The preform thus obtained was dried and recoated in slurry, this time using vacuum to ensure coverage. This process was repeated using slurries of different viscosities.

The measured weight gain of the sample after the first coating in slurry slightly increased with increasing slurry viscosity, and became constant for high-viscosity slurries. However, for the second coating, the measured weight gain was double that of the first coating for low-viscosity slurries, and increased dramatically with slurry viscosity. This is an effect of the enhanced adsorption created by the first thin film deposited on the lattice, said the researchers.

Measurements of the diameters in the lattice struts were fairly uniform and almost identical to the joint diameters. The strut diameter increased with increasing slurry viscosity, as expected, since the weight gain was higher at higher viscosity in the second coating. Through this process, the researchers demonstrated the ability to obtain a highly uniform and robust reticule structure, even starting with any irregularly shaped sample. The combination of highspeed centrifuging after each coating and the use of high-viscosity slurry for the second coating improved mechanical properties of RPCs, the researchers reported. The only difficulty they noted was the need for completing the second coating before drying the sample to prevent it from absorbing water and crashing.

SIARI SOSA

\section{Circuit Quantum Electrodynamics Used to Couple Photon with Qubit}

One of the goals in quantum physics is to develop a quantum computer in which the transistors that form logic gates to manipulate bits in a classical computer are replaced with quantum logic gates that can store and process information quantum mechanically. The fundamental units of information manipulated in a quantum computer are called quantum bits or "qubits," but implementations of the quantum logic gates are sometimes also referred to as qubits. R. Schoelkopf and the theory group of S. Girvin, both at Yale University, have conducted an experiment in which a single photon was coherently coupled to a single superconducting qubit or artificial atom consisting of about 10 billion aluminum atoms acting in concert like a single atom (see Figure 1). One way to connect qubits in a quantum computer is to make a "quantum bus" to carry information back and forth by using single photons. The work of Schoelkopf, Girvin, postdoctoral associate A. Wallraff, and graduate student D. Schuster introduces a paradigm in which quantum optics experiments can be performed in a microchip electrical circuit using microwaves instead of visible photons and lasers.

As reported in the September 9 issue of Nature (p. 162), the researchers constructed a miniaturized superconducting cavity based upon a coplanar waveguide structure of niobium on a silicon substrate in which to trap the microwave photon and force it to collide about 12 million times per second with the qubit implementation based upon a Cooper pair box. The Cooper pair box consisted of a long, thin superconducting island (several microns

For more information, see http://advertisers.mrs.org 